\title{
A Rare Syndrome in the Differential Diagnosis for Angioedema
}

\author{
Zeynep Celebi Sozenera, d, Selcan Ozguclu ${ }^{\mathrm{b}}$, Omur Aydinn ${ }^{\mathrm{a}}$, Aylin Okcu Heperc, Sadan Soyyigit ${ }^{\mathrm{a}}$, \\ Ayse Betul Sin ${ }^{\mathrm{a}}$
}

\begin{abstract}
Melkersson-Rosenthal syndrome (MRS) is a rare, non-caseating chronic granulamatous neurocutaneous disease. MRS is frequently seen at the second or third decades of life. Classic triad was comprised of the recurrent facial nerve paralysis, orofacial edema and fissured dorsal tongue (lingua plicata). It is seen in $8-25 \%$ of the cases. The most important differential diagnosis of MRS is angioedema. Here we present two cases with MRS from our clinic. The first one is an example for the classic triad and the histopathological appearance is consistent with granulomatous cheilitis. The second case has an nonspesific histopathologic examination and was diagnosed clinically.
\end{abstract}

Keywords: Angioedema; Melkersson-Rosenthal syndrome; Lingua plicata; Granulamatous cheilitis; Allergy

\section{Introduction}

Melkersson-Rosenthal syndrome (MRS) is a rare, non-caseating chronic granulamatous neurocutaneous disease [1]. Although its etiology and pathogenesis is still unknown, genetic factors, immunodeficiency, infections, atopy and stress are being accused [2]. Some researchers have considered the syndrome to be a manifestation of sarcoidosis or Crohn's disease [3]. This syndrome is more frequently seen in the second or third decade. Classic triad comprised of the recurrent facial nerve paralysis, orofacial edema and fissured dorsal tongue (lingua plicata) is not often. Manifestations may develop at dif-

Manuscript accepted for publication October 18, 2013

aDepartment of Chest Diseases, Division of Immunology and Allergy, School of Medicine, Ankara University, Ankara, Turkey

bDepartment of Internal Diseases, Division of Immunology and Allergy, School of Medicine, Ankara University, Ankara, Turkey

'Department of Pathology, School of Medicine, Ankara University, Ankara, Turkey

${ }^{\mathrm{d} C o r r e s p o n d i n g ~ A u t h o r: ~ Z e y n e p ~ C e l e b i ~ S o z e n e r, ~ D e p a r t m e n t ~ o f ~ C h e s t ~ D i s-~}$ eases, Division of Immunology and Allergy, School of Medicine, Ankara University, Ankara 06620, Turkey. Email: zeynepsozener@gmail.com

doi: http://dx.doi.org/10.14740/jmc1543w ferent times. The presence of one or two symptoms associated with granulamatous cheilitis in the biopsy material is adequate for diagnosis $[2,4]$. Here we present two cases with MRS from our clinic.

\section{Case Reports}

\section{Case 1}

A 39-year-old man admitted to our clinic with recurrent lip edema that started 3 years ago. The patient reported persistent swelling at his upper left lip for 6 months and worsening in the last 2 days. Before admission to our hospital, he received antihistamine and steroid treatments with angioedema diagnosis. For every 3- to 4-month intervals, his lip swallowed and improved with antihistaminic drug. But for 6 months, despite taking antihistamine and steroid treatment, his edema became persistent and worsened with cold (Fig. 1). He had a previous history of facial paralysis 16 years ago. The patient denied tongue, throat, and extremity swelling as well as edema on his eyes. He did not suffer from dyspnea and hoarseness. He also did not have drug or food allergy. He noted no pruritus, burning, or urticaria. His physical examination revealed swollen lips, a fissured tongue and an erased left nasolabial sulcus (Fig. $2)$. His biochemical markers including whole blood count, ASO, C-reactive protein, $\operatorname{IgG}, \operatorname{IgA}, \operatorname{IgM}$ antibodies, thyroid

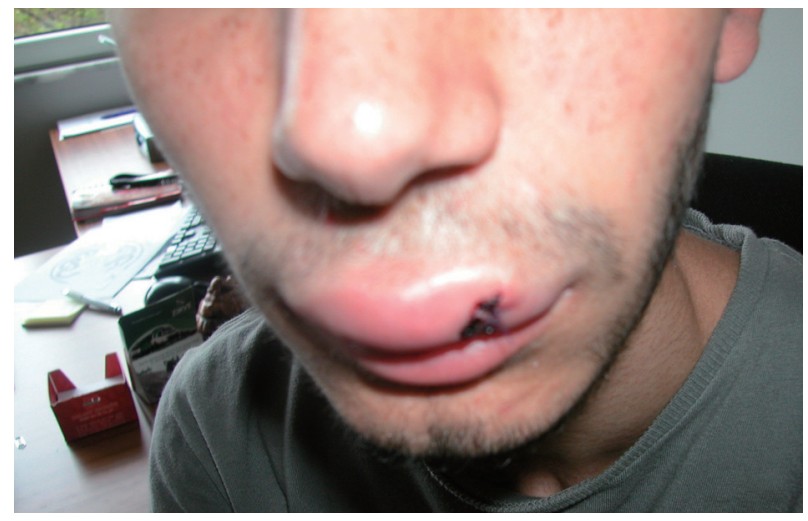

Figure 1. Marked edema on the upper lip. 


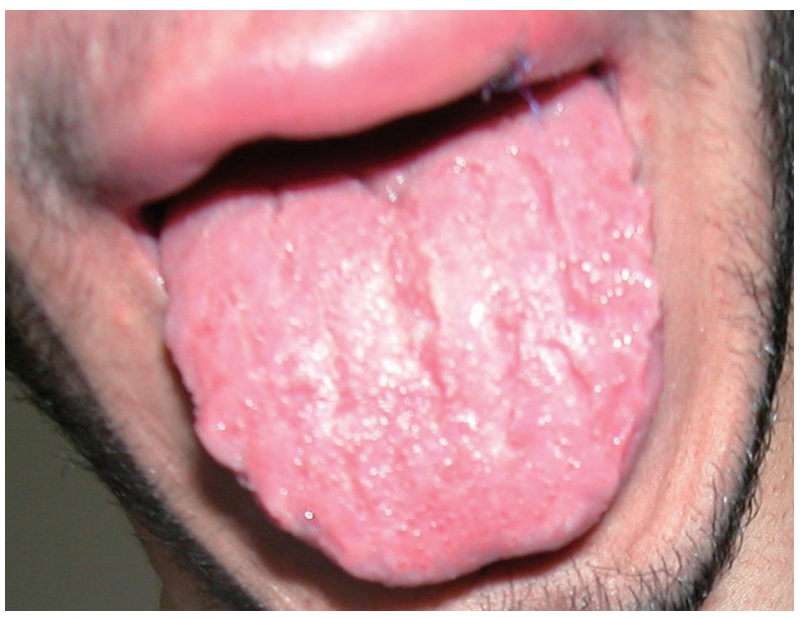

Figure 2. Fissured tongue.

function tests, fecal and urine examinations were all normal. In addition, $\mathrm{C} 1$-esterase inhibitor level and function, C3 and C4 complement levels were also found to be normal. Since cold increased his symptoms, we performed ice cube test. The result was negative. After the normal laboratory data, we recede angioedema as a differential diagnosis. Patient was consulted to the dermatology clinic. A biopsy was taken from his swollen lip mucosa and histopathological appearance was consistent with granulomatous cheilitis (Fig. 3a, b). In conclusion, we diagnosed him as MRS according to clinical and biopsy findings (labial swelling, fissured tongue, facial paralysis history and granulomatous cheilitis). His symptoms improved with intralesional steroid injection treatment.

\section{Case 2}

A 20-year-old man was seen in our clinic with persistent upper lip edema without pain, burning and local pruritus for 2 years. No causative factors, such as food, drug, latex, physical, chemical or emotional conditions could be identified. He had no edema on his eyes, throat and tongue. He did not suffer from dyspnea and hoarseness. On his physical examination, swelling on his upper lip and a fissured tongue were noticed. The patient had been treated with corticosteroids with no improvement. Laboratory investigations including complete blood count, biochemical markers, thyroid function tests, ASO, C-reactive protein, $\mathrm{C} 3$ and $\mathrm{C} 4$ complement levels, $\mathrm{C} 1$ inhibitor level and function, urine and fecal examinations were within the normal reference ranges. On consideration of the differential diagnosis, we suspected granulomatous disease and the patient was referred to dermatology clinic for an upper lip mucosal biopsy. The histopathological examination revealed nonspecific perivascular dermatitis characterized with achanthosis and hyperkeratosis. Because the result did not rule out MRS, we decided to repeat the skin biopsy. Although the second biopsy result was also nonspecific, clinical presentation led us to the diagnosis of MRS. Intralesional steroid injections were administered weekly for 3 weeks with $100 \mathrm{mg}$ tetracycline treatment. After 2 months, his lip improved $70 \%$ and he did not suffer from swelling for 1.5 months.

\section{Discussion}

In differential diagnosis of angioedema, in addition to usual causes, allergists need to consider MRS [5]. MRS was first described as the presence of recurrent facial paralysis and edema in 1928 [1, 2, 4]. In 1931, the component of lingua plicata was added. MRS is frequently seen at the second or third decades of life. Autosomal dominant inheritance is documented. Responsible gene is at the short arm of the ninth chromosome [1]. Classical triad is seen in $8-25 \%$ of the cases as well as in our first patient [1]. Patients may exhibit all components of the triad simultaneously or at different times [6].

Orofacial edema is the most frequent finding and is seen in $80-100 \%$ of the cases $[1,2,4]$. It is not painful, frequently unilateral and non-pitting. Generally the upper lip is involved. Cheek, palate, tongue, pharynx, larynx and periorbital region are rarely affected. Clinically, it is similar to angioedema; however, it is more persistent than angioedema, and it does not improve with antihistamines. It can lead to fibrosis of the involved tissues [6]. In the beginning, edema appears intermittently but it may be continuous later as in our case. Monosymptomatic cases with perioral edema are named as granulomatous cheilitis [4].

MRS can be associated with underlying disorders, such as sarcoidosis, and is part of the spectrum of orofacial granulomatosis [7]. This entity includes cheilitis granulomatosa, sarcoidosis or Crohn's disease. Labial involvement alone is referred as cheilitis granulomatosa. A clinical aspect of Crohn's disease, which is known as orofacial Crohn's disease, may present with granulomatous lesions of the face and the oral cavity. Sarcoidosis also presents with facial granulomatous inflammation. Histologically, these disorders display dermal edema and mixed perivascular inflammation [3].

MRS-related facial paralysis is found up to $50-60 \%$ of the cases. It may be recurrent in $3-11 \%$ of them. Facial paralysis develops many years before or after orofacial edema [2, 4]. In our first case, he had facial paralysis 16 years before orofacial edema arises. Peripheral facial paralysis can be unilateral or bilateral, as well as being partial or complete [6].

Fissured tongue is present in $30-35 \%$ of the cases. Despite the other components of the triad, fissured tongue is also present in normal population at $5 \%$. So, people with isolated fissured tongue cannot be diagnosed as MRS $[1,2,4]$.

Only $8-25 \%$ of patients with this disorder represent the complete triad. Therefore, cheilitis granulomatosa was used to describe the monosymptomatic or oligosymptomatic form of MRS [3]. Histopathologic evidence has been suggested to be not necessary and the diagnosis is based on clinical symptoms [6]. However, biopsy may help to diagnose MRS. Presence of one or two symptoms with granulomatous chellitis in histopathological examination is enough for diagnosis. Characteristic histopathological features of MRS are lymphedema, non-caseating epitheloid cell granulomas, multinucleated Langerhans-type giant cells, perivascular mononuclear inflam- 

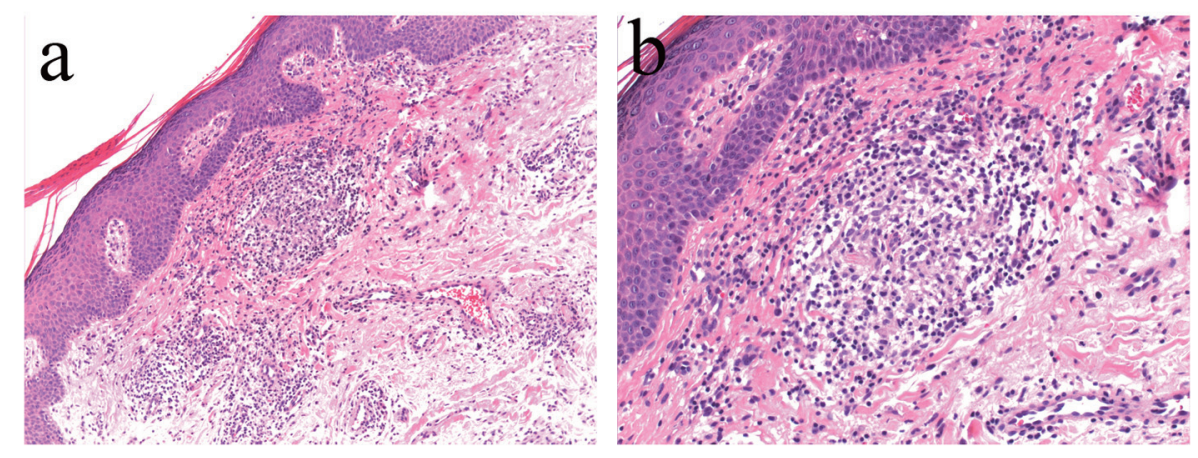

Figure 3. (a) There is a perivascular inflammatory infiltrate, beneath the squamous epithelium of mucosa (H\&E, $\times 100)$. (b) Closer view of the mucosal superficial perivascular inflammation. There is a subtle granuloma, intermixed with lymphocytes and plasma cells $(H \& E, \times 200)$.

matory infiltration and fibrosis [6]. Non-caseous perivascular granulomas are very important for diagnosis but their absence does not rule out the diagnosis as shown in our second patient [4].

Causes of recurrent facial paralysis include allergic diseases with orofacial angioedema, dental problems, hypothyroidism, superior vena cava syndrome, lymphangioma, erysipelas, lymphoma, and herpes simplex labialis and all should be considered in the differential diagnosis $[1,2]$. The most important differential diagnosis is angioedema. MRS may usually be distinguished from angioedema because of the chronic nature of orofacial swelling and usually normal complement titers [7]. After clinical and laboratory examination, we eliminated the other causes for diagnosis of MRS in our cases.

Treatment in MRS is possible but recurrences are unavoidable [2]. Spontaneous remission of MRS has also been reported [5]. Steroids are used in the medical treatment to resolve the edema. Intralesional steroid injection treatments are as effective as systemic steroids [5]. Clofazimine, minoxicillin, MTX, danazol, dapsone, sulfasalazine, penicillin, tetracycline, and clindamycin have also been tried as a treatment choice $[1$, $2,4,8]$. Clofazimine and minoxicillin are found to be as effective as steroids to regress the orofacial edema and granuloma forming when used together or solely [1]. Infliximab has been shown to be effective in the treatment of orofacial Crohn's disease. Because of the similarities between Crohn's disease and MRS, infliximab was tried to treat patients with MRS [3], and it has shown successful results after treatment. Radiotherapy was also tried as a treatment choice but it was not found to be beneficial [2]. Another reported procedure is the use of helium-neon laser radiation treatment. It was reported that patients with disease duration less than 4 years had a significant benefit from this treatment [9]. Surgical techniques like chelioplasty or facial nerve decompression should be preferred when medical treatment fails $[1,2]$. We administered intralesional steroids in our first case and intralesional steroids combined with oral tetracycline in the second. Both of them improved with the treatment.

In a retrospective case series, it was reported that the MRS may present over the course of the life span, and may require several years of observation to be diagnosed [10]. Therefore, MRS should be considered as a differential diagnosis for an- gioedema with the presence of persistent orofacial edema and recurrent facial paralysis. With these case reports, we wanted to remind this rare diagnosis in terms of pseudoangioedema cases.

\section{Disclosure}

The authors declare they have no financial or any conflicts of interest.

\section{References}

1. Melek H, Koken R, Bukulmez A. Melkersson-Rosenthal Sendromu: Bir Olgu Sunumu. Guncel Pediatri. 2007;5:82-84.

2. Aktar F, Sal E, Acikgoz M. Melkersson-Rosenthal Sendromu: Bir Olgu Sunumu. Van Tip Dergisi. 2011;18:5760 .

3. Kakimoto C, Sparks C, White AA. Melkersson-Rosenthal syndrome: a form of pseudoangioedema. Ann Allergy Asthma Immunol. 2007;99(2):185-189.

4. Senel E, Gulec T. Melkersson-Rosenthal Syndrome. Successfully Treated with Intralesional Corticosteroid; A case Report. Turk J Dermatol. 2009;3:16-18.

5. Perez-Calderon R, Gonzalo-Garijo MA, Chaves A, de Argila D. Cheilitis granulomatosa of Melkersson-Rosenthal syndrome: treatment with intralesional corticosteroid injections. Allergol Immunopathol (Madr). 2004;32(1):3638.

6. Ozgursoy OB, Karatayli Ozgursoy S, Tulunay O, Kemal O, Akyol A, Dursun G. Melkersson-Rosenthal syndrome revisited as a misdiagnosed disease. Am J Otolaryngol. 2009;30(1):33-37.

7. Masson F, Barete S, Fremeaux-Bacchi V, Szpirglas H, Agbo-Godeau S, Chosidow O, Piette JC, et al. Melkersson-Rosenthal syndrome and acquired $\mathrm{C} 1$ inhibitor deficiency. Dermatology. 2008;217(2):114-120.

8. Talabi OA. Melkerssons-Rosenthal syndrome: a case report and review of the literature. Niger J Clin Pract. $2011 ; 14(4): 477-478$. 
9. Banks T, Gada S. A comprehensive review of current treatments for granulomatous cheilitis. Br J Dermatol. 2012;166(5):934-937.
10. Elias MK, Mateen FJ, Weiler CR. The MelkerssonRosenthal syndrome: a retrospective study of biopsied cases. J Neurol. 2013;260(1):138-143. 